\title{
SURFACES WITH INFLECTION POINTS IN EUCLIDEAN 4-SPACE
}

\author{
Reiko Aiyama and Kazuo Akutagawa*
}

\begin{abstract}
For a surface in the Euclidean 4-space, we prove a reduction theorem for the codimension of a surface all whose points are inflection points.
\end{abstract}

\section{Introduction}

The curvature ellipse is much interested in the study of a surface $M$ in the Euclidean 4-space $\mathbf{R}^{4}$ (cf. $\left.[8,9,4]\right)$. At a point $p \in M$, the curvature ellipse $\mathscr{E}_{p}$ is defined by the image $\left\{\Pi(v, v) \in T_{p}^{\perp} M\left|v \in T_{p} M,\right| v \mid=1\right\}$, in the normal space $T_{p}^{\perp} M$, of the unit circle in the tangent plane $T_{p} M$ under the second fundamental form $\Pi$. If the curvature ellipse $\mathscr{E}_{p}$ degenerates to a segment contained in a straight line passing through $\mathbf{0}_{p}$ of $T_{p}^{\perp} M$, we say that $p$ is an inflection point. A sufficient and necessary condition for $p$ being an inflection point is that there exists a unit normal vector $v_{p} \in T_{p}^{\perp} M$ such that the $v$-component $\langle\Pi, v\rangle$ of $\Pi$ at $p$ vanishes. In particular, if $M$ lies an affine 3 -space in $\mathbf{R}^{4}$, then all points are inflection points. On the other hand, the converse does not hold (e.g. Example 5.2, (ii)). Lane [7] proved that if the surface is exclusively made of inflection points, then it is locally either a developable surface or lies in a 3-space (cf. Little [8]). In this paper, we present the following reduction theorem.

THEOREM 1. Let $X$ be a conformal immersion from a connected Riemann surface $S$ into $\mathbf{R}^{4}$. Assume that the Gauss curvature $K$ does not vanish anywhere. If all points of $S$ are inflection points, then the surface $X(S)$ lies in an affine 3-space in $\mathbf{R}^{4}$.

In order to prove this theorem, we introduce a new complex-valued local invariant $\Lambda$ in Section 2. For the resultant $\Delta_{p}$ of $X$ at $p \in S$ and the normal

1991 Mathematics Subject Classification. Primary 53A05, Secondary 53B25.

Key words and phrases. curvature ellipses, inflection points, surfaces in Euclidean 4-space, Gauss maps.

* supported in part by the Grant-in-Aid for Challenging Exploratory Research, Japan Society for the Promotion of Science, No. 24654009.

Received April 30, 2013; revised August 27, 2013. 
curvature $K_{N}(p), \Lambda(p)$ satisfies that

$$
4 \Delta_{p}=\left(K_{N}(p)\right)^{2}-4|\Lambda(p)|^{2} .
$$

The local invariant $\Delta_{p}$ was considered in $[8,9,4]$, in order to study of the curvature ellipse $\mathscr{E}_{p}$. The sign of $\Delta_{p}$ determines the position of $\mathscr{E}_{p}$ in $T_{p}^{\perp} M$, that is, whether the origin $\mathbf{0}_{p}$ of $T_{p}^{\perp} M$ lies inside of $\mathscr{E}_{p}$ or outside of $\mathscr{E}_{p}$ or on $\mathscr{E}_{p}$ (Lemma 2, cf. [8, Section 2]). However, the relation between $\Delta_{p}$ and curvatures of $M$ is not clear since $\Delta_{p}$ is a polynomial of degree 4 with respect to the components of second fundamental form $\Pi$. On the other hand, the invariant $\Lambda(p)$ is a quadratic polynomial with respect to the components of the mean curvature vector and the Hopf differential. Hence, the criterion on the position of $\mathscr{E}_{p}$ in $T_{p}^{\perp} M$ is explicitly expressed in terms of cuvatures of $X(S)$.

In Section 2, we recall the definition of curvature ellipses $\mathscr{E}_{p}$ and the invariant $\Delta_{p}$. Then we introduce the invariant $\Lambda$. Moreover, we give another simple proof of the above fact (i.e., Lemma 2) by using $\Lambda$ and $K_{N}$. In Section 3, we represent $\Lambda$ in terms of the Gauss maps. In Section 4, we prove Theorem 1. In Section 5, we give some examples of surfaces in $\mathbf{R}^{4}$.

\section{Curvature ellipses}

We prepare the terminologies following [8] (see also [4]).

Let $S$ be a connected Riemann surface and $X: S \rightarrow \mathbf{R}^{4}$ a conformal immersion. From now on, we identify locally $S$ with $X(S)\left(\subset \mathbf{R}^{4}\right)$ via the immersion $X$. Let $\left\{e_{1}, e_{2}, e_{3}, e_{4}\right\}$ denote an orthonormal frame on an open neighborhood of $S$, chosen $e_{1}$ and $e_{2}$ are tangent vectors to $S$ with the frame $\left\{e_{1}, e_{2}\right\}$ agreeing with the orientation of $T_{p} S$, and chosen so that $e_{3}$ and $e_{4}$ are normal to the surface with the frame $\left\{e_{1}, e_{2}, e_{3}, e_{4}\right\}$ agreeing with a fixed orientation of $\mathbf{R}^{4}$. As usual, define the dual forms $\omega_{A}=d X \cdot e_{A}$ and the connection forms $\omega_{A}^{B}=d e_{A} \cdot e_{B}$. The indices $A, B$ run from 1 to 4 . Then we have the structure equations:

$$
\omega_{A}^{B}=-\omega_{B}^{A}, \quad d \omega_{A}=\sum_{B=1}^{4} \omega_{A}^{B} \wedge \omega_{B}, \quad d \omega_{A}^{B}=\sum_{C=1}^{4} \omega_{A}^{C} \wedge \omega_{C}^{B} .
$$

Since $\omega_{3}=\omega_{4}=0$ on $S$, by the Cartan Lemma, we obtain the functions $h_{i j}^{\alpha}$ such that $\omega_{i}^{\alpha}=\sum_{j=1}^{2} h_{i j}^{\alpha} \omega_{j}$. The indices $i, j$ run from 1 to 2 , and $\alpha, \beta$ run from 3 to 4 . We have the symmetry $h_{i j}^{\alpha}=h_{j i}^{\alpha}$. The second fundamental form $\Pi$ of the surface is

$$
\Pi=\left(d^{2} X \cdot e_{3}\right) e_{3}+\left(d^{2} X \cdot e_{4}\right) e_{4}=\sum_{\alpha=3}^{4} \sum_{i, j=1}^{2} h_{i j}^{\alpha} \omega_{i} \omega_{j} e_{\alpha} .
$$

The Gauss curvature $K$ is defined by the formula

$$
d \omega_{1}^{2}=-K \omega_{1} \wedge \omega_{2} .
$$


The normal curvature $K_{N}$ is also defined by the formula

$$
d \omega_{3}^{4}=-K_{N} \omega_{1} \wedge \omega_{2} \text {. }
$$

Both the Gauss curvature $K$ and the normal curvature $K_{N}$ are described in terms of the components $h_{i j}^{\alpha}$ :

$$
\begin{aligned}
& K=h_{11}^{3} h_{22}^{3}-\left(h_{12}^{3}\right)^{2}+h_{11}^{4} h_{22}^{4}-\left(h_{12}^{4}\right)^{2}, \\
& K_{N}=\left(h_{11}^{3}-h_{22}^{3}\right) h_{12}^{4}-\left(h_{11}^{4}-h_{22}^{4}\right) h_{12}^{3} .
\end{aligned}
$$

For a given point $p \in S$, consider the unit circle $S_{p}^{1}$ in $T_{p} S$ parametrized by the angle $\theta$. We call the following map $\boldsymbol{\eta}$ from $S_{p}^{1}$ to the normal space $T_{p}^{\perp} S$ the normal curvature vector. Denote by $\gamma_{\theta}$ the unit-speed curve on $S$ satisfying $\gamma_{\theta}(0)=p$ and $\gamma_{\theta}^{\prime}(0)=\xi_{\theta}=\cos \theta e_{1}+\sin \theta e_{2}$, and define $\boldsymbol{\eta}(\theta)=\boldsymbol{\eta}\left(\xi_{\theta}\right)$ by the normal part of $\gamma_{\theta}^{\prime \prime}(0)$. Then we obtain that

$$
\begin{aligned}
\boldsymbol{\eta}(\theta) & =\sum_{\alpha=3}^{4} \sum_{i, j=1}^{2} h_{i j}^{\alpha} \omega_{i}\left(\xi_{\theta}\right) \omega_{j}\left(\xi_{\theta}\right) e_{\alpha} \\
& =\left(\begin{array}{ll}
e_{3} & e_{4}
\end{array}\right)\left(\begin{array}{l}
h_{11}^{3} \cos ^{2} \theta+2 h_{12}^{3} \cos \theta \sin \theta+h_{22}^{3} \sin ^{2} \theta \\
h_{11}^{4} \cos ^{2} \theta+2 h_{12}^{4} \cos \theta \sin \theta+h_{22}^{4} \sin ^{2} \theta
\end{array}\right) \\
& =\left(\begin{array}{ll}
e_{3} & e_{4}
\end{array}\right)\left(\begin{array}{l}
\frac{1}{2}\left(h_{11}^{3}+h_{22}^{3}\right)+\frac{1}{2}\left(h_{11}^{3}-h_{22}^{3}\right) \cos 2 \theta+h_{12}^{3} \sin 2 \theta \\
\frac{1}{2}\left(h_{11}^{4}+h_{22}^{4}\right)+\frac{1}{2}\left(h_{11}^{4}-h_{22}^{4}\right) \cos 2 \theta+h_{12}^{4} \sin 2 \theta
\end{array}\right)
\end{aligned}
$$

Recall that the mean curvature vector $\boldsymbol{H}$ is given by

$$
\boldsymbol{H}=\frac{1}{2}\left(h_{11}^{3}+h_{22}^{3}\right) e_{3}+\frac{1}{2}\left(h_{11}^{4}+h_{22}^{4}\right) e_{4} .
$$

Then we have

$$
\boldsymbol{\eta}(\theta)-\boldsymbol{H}=\left(\begin{array}{ll}
e_{3} & e_{4}
\end{array}\right) \mathscr{H}\left(\begin{array}{c}
\cos 2 \theta \\
\sin 2 \theta
\end{array}\right), \quad \text { where } \mathscr{H}=\left(\begin{array}{cc}
\frac{1}{2}\left(h_{11}^{3}-h_{22}^{3}\right) & h_{12}^{3} \\
\frac{1}{2}\left(h_{11}^{4}-h_{22}^{4}\right) & h_{12}^{4}
\end{array}\right)
$$

The normal curvature $K_{N}$ coincides with $2 \operatorname{det}(\mathscr{H})$. When $K_{N}$ is not zero at $p$, the locus $\mathscr{E}_{p}$ of $\boldsymbol{\eta}(\theta)$ is an ellipse centered at $\boldsymbol{H}$ in $T_{p}^{\perp} S$. So we call the locus $\mathscr{E}_{p}$ the curvature ellipse at $p$. When $K_{N}$ is zero at $p$, the curvature ellipse $\mathscr{E}_{p}$ is a segment.

At a point $p$ in $S$, if the origin $\mathbf{0}_{p}$ of $T_{p}^{\perp} S$ lies outside the curvature ellipse $\mathscr{E}_{p}$, then the point $p$ is said to be hyperbolic. The point $p$ of $S$ is said to be elliptic if $\mathbf{0}_{p}$ lies inside $\mathscr{E}_{p}$, and the point $p$ of $S$ is said to be parabolic if $\mathbf{0}_{p}$ lies on $\mathscr{E}_{p}$. (In [11], the hyperbolic points are said to be convex and the elliptic points are said to be aconvex.) When $\mathscr{E}_{p}$ degenerates to a segment contained in 
a straight line passing through $\mathbf{0}_{p}$, the point $p$ of $M$ is said to be an inflection point. At an inflection point $p$ in $S, K_{N}=0$ at $p$. Moreover, we can choose a unit normal vector $\tilde{e}_{3} \in T_{p}^{\perp} S$ such that the components of the second fundamental form with respect to $\tilde{e}_{3}$ are zero, that is,

$$
d^{2} X \cdot \tilde{e}_{3}=\sum_{i, j=1}^{2} \tilde{h}_{i j}^{3} \omega_{i} \omega_{j}=\mathbf{0} .
$$

The last condition is a necessary and sufficient condition for that $p$ is an inflection point.

The resultant $\Delta_{p}$ of $X$ at $p$ is defined by

$$
\Delta_{p}=\frac{1}{4}\left|\begin{array}{cccc}
h_{11}^{3} & 2 h_{12}^{3} & h_{22}^{3} & 0 \\
h_{11}^{4} & 2 h_{12}^{4} & h_{22}^{4} & 0 \\
0 & h_{11}^{3} & 2 h_{12}^{3} & h_{22}^{3} \\
0 & h_{11}^{4} & 2 h_{12}^{4} & h_{22}^{4}
\end{array}\right|,
$$

which is the resultant of the two polynomials $h_{11}^{3} x^{2}+2 h_{12}^{3} x y+h_{22}^{3} y^{2}$ and $h_{11}^{4} x^{2}+2 h_{12}^{4} x y+h_{22}^{4} y^{2}$. By the resultant $\Delta_{p}$, we can distinguish the position of $\mathscr{E}_{p}$ in $T_{p}^{\perp} M$ as follows:

Lemma 2 ([8], [9]). At a point $p$ of $S$, assume that $K_{N} \neq 0$.

(i) $p$ is a hyperbolic point if and only if $\Delta_{p}<0$.

(ii) $p$ is a parabolic point if and only if $\Delta_{p}=0$.

(iii) $p$ is an elliptic point if and only if $\Delta_{p}>0$.

Set $h^{\alpha}=\frac{1}{2}\left(h_{11}^{\alpha}+h_{22}^{\alpha}\right)$ and $\varphi^{\alpha}=\frac{1}{2}\left(h_{11}^{\alpha}-h_{22}^{\alpha}\right)-i h_{12}^{\alpha}(\alpha=3,4)$, where $\boldsymbol{i}$ denotes the imaginary unit. Then we have $\boldsymbol{H}=h^{3} e_{3}+h^{4} e_{4}, K=\left(h^{3}\right)^{2}+\left(h^{4}\right)^{2}-\left|\varphi^{3}\right|^{2}-$ $\left|\varphi^{4}\right|^{2}$ and $K_{N}=2 \operatorname{Im}\left(\varphi^{3} \overline{\varphi^{4}}\right)$. Moreover, we set

$$
\Lambda=-h^{3} \varphi^{4}+h^{4} \varphi^{3} \text {. }
$$

Then, we have the following lemma by a straightforward computation.

Lemma 3. At a point $p$ of $S$,

$$
4 \Delta_{p}=\left(K_{N}(p)\right)^{2}-4|\Lambda(p)|^{2}
$$

Remark 4. We can write

$$
d\left(e_{1}-\boldsymbol{i} e_{2}\right) \cdot\left(e_{3}+\boldsymbol{i} e_{4}\right) \wedge d\left(e_{1}-\boldsymbol{i} e_{2}\right) \cdot\left(e_{3}-i e_{4}\right)=-2 \boldsymbol{i} \Lambda \phi \wedge \bar{\phi},
$$

where $\phi=\omega_{1}+\boldsymbol{i} \omega_{2}$.

The normal curvature vector $\boldsymbol{\eta}(\theta)$ at $p \in S$ is given by

$$
\boldsymbol{\eta}(\theta)=\left(\begin{array}{ll}
e_{3} & e_{4}
\end{array}\right)\left(\begin{array}{c}
h^{3}+\operatorname{Re}\left(\varphi^{3} e^{i 2 \theta}\right) \\
h^{4}+\operatorname{Re}\left(\varphi^{4} e^{i 2 \theta}\right)
\end{array}\right),
$$


and

$$
\frac{d \boldsymbol{\eta}}{d \theta}=\left(\begin{array}{ll}
e_{3} & e_{4}
\end{array}\right)\left(\begin{array}{c}
-2 \operatorname{Im}\left(\varphi^{3} e^{i 2 \theta}\right) \\
-2 \operatorname{Im}\left(\varphi^{4} e^{i 2 \theta}\right)
\end{array}\right)
$$

Proof of Lemma 2. We give here a different proof from that in $[8$, Section 2]. When $\mathbf{0}_{p}$ lies outside the curvature ellipse $\mathscr{E}_{p}$, there exist $\theta_{1}, \theta_{2} \in[0, \pi)$ $\left(\theta_{1} \neq \theta_{2}\right)$ such that the tangent vectors $\frac{d \boldsymbol{\eta}}{d \theta}\left(\theta_{i}\right)$ of $\mathscr{E}_{p}$ is a scalar multiplication of the position vectors $\boldsymbol{\eta}\left(\theta_{i}\right)(i=1,2)$. This implies that the following equation for $\theta$ must have two distinct solutions:

$$
0=\operatorname{det}\left(\boldsymbol{\eta}(\theta) \quad-\frac{1}{2} \frac{d \boldsymbol{\eta}}{d \theta}\right)=\left|\begin{array}{ll}
h^{3}+\operatorname{Re}\left(\varphi^{3} e^{i 2 \theta}\right) & \operatorname{Im}\left(\varphi^{3} e^{i 2 \theta}\right) \\
h^{4}+\operatorname{Re}\left(\varphi^{4} e^{i 2 \theta}\right) & \operatorname{Im}\left(\varphi^{4} e^{i 2 \theta}\right)
\end{array}\right| .
$$

This equation implies that $h^{3}+\varphi^{3} e^{i 2 \theta}$ and $h^{4}+\varphi^{4} e^{i 2 \theta}$ lie on the same line through the origin in the complex plane. We then obtain

$$
\begin{aligned}
0 & =\operatorname{Im}\left\{\left(h^{3}+\varphi^{3} e^{i 2 \theta}\right) \overline{\left(h^{4}+\varphi^{4} e^{i 2 \theta}\right)}\right\} \\
& =\operatorname{Im}\left\{\left(-h^{3} \varphi^{4}+h^{4} \varphi^{3}\right) e^{i 2 \theta}\right\}+\frac{1}{2} K_{N} .
\end{aligned}
$$

Then, we have $|\Lambda|=\left|-h^{3} \varphi^{4}+h^{4} \varphi^{3}\right|>\frac{1}{2}\left|K_{N}\right|$.

When $\mathbf{0}_{p} \in \mathscr{E}_{p}$, there exists only one $\theta \in \mathbf{R} / \pi \mathbf{Z}$ satisfying (4). Then, we have $|\Lambda|=\left|-h^{3} \varphi^{4}+h^{4} \varphi^{3}\right|=\frac{1}{2}\left|K_{N}\right|$.

When $\mathbf{0}_{p}$ lies inside $\mathscr{E}_{p}$, there exists no solution of the above equation (4). Then, we have $|\Lambda|=\left|-h^{3} \varphi^{4}+h^{4} \varphi^{3}\right|<\frac{1}{2}\left|K_{N}\right|$.

LEMma 5. At a point $p$ of $S$, assume that $K_{N}=0$.

(I) The curvature ellipse $\mathscr{E}_{p}$ consists of only one point if and only if $\varphi^{3}=$ $\varphi^{4}=0$ at $p . \quad$ In this case, the origin $\mathbf{0}_{p}$ of $T_{p} S$ lies on $\mathscr{E}_{p}$ if and only if $\boldsymbol{H}=\mathbf{0}$ at $p$.

(II) The curvature ellipse $\mathscr{E}_{p}$ is a segment (which is not only one point) if and only if $\varphi^{3} \neq 0$ or $\varphi^{4} \neq 0$ at $p$.

(i) The origin $\mathbf{0}_{p}$ of $T_{p} S$ lies on the segment as the curvature ellipse $\mathscr{E}_{p}$ if and only if $\Lambda=0,\left|h^{3}\right| \leqq\left|\varphi^{3}\right|$ and $\left|h^{4}\right| \leqq\left|\varphi^{4}\right|$ at $p$.

(ii) The origin $\mathbf{0}_{p}$ of $T_{p} S$ lies at the end points of the segment as the curvature ellipse $\mathscr{E}_{p}$ if and only if $\Lambda=0,\left|h^{3}\right|=\left|\varphi^{3}\right|$ and $\left|h^{4}\right|=\left|\varphi^{4}\right|$ at $p$.

Proof. (I) It follows from the equation (2).

(II) When $\mathbf{0}_{p}$ lies on the segment $\mathscr{E}_{p}$, there exists $\theta \in \mathbf{R} / \pi \mathbf{Z}$ such that $\boldsymbol{\eta}(\theta)=\mathbf{0}$, and hence $\operatorname{Re}\left(\varphi^{\alpha} e^{i 2 \theta}\right)=-h^{\alpha}(\alpha=3,4)$. This implies that $\left|\varphi^{\alpha}\right| \geqq\left|h^{\alpha}\right|$, and hence 


$$
\begin{aligned}
0 & =\frac{1}{2} K_{N}=\operatorname{Im}\left(\varphi^{3} e^{i 2 \theta} \overline{\varphi^{4} e^{i 2 \theta}}\right) \\
& =-\operatorname{Re}\left(\varphi^{3} e^{i 2 \theta}\right) \operatorname{Im}\left(\varphi^{4} e^{i 2 \theta}\right)+\operatorname{Im}\left(\varphi^{3} e^{i 2 \theta}\right) \operatorname{Re}\left(\varphi^{4} e^{i 2 \theta}\right) \\
& =h^{3} \operatorname{Im}\left(\varphi^{4} e^{i 2 \theta}\right)-h^{4} \operatorname{Im}\left(\varphi^{3} e^{i 2 \theta}\right) \\
& =-\operatorname{Im}\left\{\left(-h^{3} \varphi^{4}+h^{4} \varphi^{3}\right) e^{i 2 \theta}\right\}
\end{aligned}
$$

Furthermore, we have

$$
\begin{aligned}
\left(-h^{3} \varphi^{4}+h^{4} \varphi^{3}\right) e^{i 2 \theta} & =\operatorname{Re}\left\{\left(-h^{3} \varphi^{4}+h^{4} \varphi^{3}\right) e^{i 2 \theta}\right\} \\
& =-h^{3} \operatorname{Re}\left(\varphi^{4} e^{i 2 \theta}\right)+h^{4} \operatorname{Re}\left(\varphi^{3} e^{i 2 \theta}\right)=0 .
\end{aligned}
$$

Then, we obtain that $\Lambda=-h^{3} \varphi^{4}+h^{4} \varphi^{3}=0$.

Conversely, assume that $\Lambda=0,\left|h^{3}\right| \leqq\left|\varphi^{3}\right|$ and $\left|h^{4}\right| \leqq\left|\varphi^{4}\right|$ at $p$. There exists $\theta_{\alpha} \in \mathbf{R} / \pi \mathbf{Z}$ satisfying $\operatorname{Re}\left(\varphi^{\alpha} e^{i 2 \theta_{\alpha}}\right)=-h^{\alpha}$. The equation $-h^{3} \varphi^{4}+h^{4} \varphi^{3}=0$ implies the existence of $w \in \mathbf{C}$ satisfying $\left(\begin{array}{l}h^{3} \\ h^{4}\end{array}\right)=w\left(\begin{array}{c}\varphi^{3} \\ \varphi^{4}\end{array}\right)$, and hence $\arg \varphi^{3}=$ $\arg \varphi^{4}\left(=: \theta_{0}\right)$. Hence, we have $\left|\varphi^{\alpha}\right| \cos \left(\theta_{0}+2 \theta_{\alpha}\right)=|w|\left|\varphi^{\alpha}\right|$, and then $\theta_{3}=$ $\theta_{4}(=: \theta)$. This gives that $\boldsymbol{\eta}(\theta)=\mathbf{0}$. Now we can conclude the assersion (i).

When $\mathbf{0}_{p}$ lies at the end points of the segment $\mathscr{E}_{p}$, there exists $\theta \in \mathbf{R} / \pi \mathbf{Z}$ such that $\boldsymbol{\eta}(\theta)=\mathbf{0}$ and $\frac{d \boldsymbol{\eta}}{d \theta}=\mathbf{0}$. Hence, we have $\varphi^{\alpha} e^{i 2 \theta}=-h^{\alpha}$, and then $\Lambda=$ $-h^{3} \varphi^{4}+h^{4} \varphi^{3}=0$ and $\left|\varphi^{\alpha}\right|=\left|h^{\alpha}\right|$.

Conversely, assume that $\Lambda=0$ and $\left|\varphi^{\alpha}\right|=\left|h^{\alpha}\right|$ at $p$. Then we can get $\theta \in \mathbf{R} / \pi \mathbf{Z}$ satisfying $\boldsymbol{\eta}(\theta)=\frac{d \boldsymbol{\eta}}{d \theta}=\mathbf{0}$ similarly to the above.

Little [8] has also proved the following equivalent condition on inflection points. In the following theorem,

$$
\mathscr{S}=\left(\begin{array}{cc}
\left|\begin{array}{cc}
h_{11}^{3} & h_{12}^{3} \\
h_{11}^{4} & h_{12}^{4}
\end{array}\right| & \frac{1}{2}\left|\begin{array}{cc}
h_{11}^{3} & h_{22}^{3} \\
h_{11}^{4} & h_{22}^{4}
\end{array}\right| \\
\frac{1}{2}\left|\begin{array}{ll}
h_{11}^{3} & h_{22}^{3} \\
h_{11}^{4} & h_{22}^{4}
\end{array}\right| & \left|\begin{array}{ll}
h_{12}^{3} & h_{22}^{3} \\
h_{12}^{4} & h_{22}^{4}
\end{array}\right|
\end{array}\right) .
$$

We remark that $\Delta=\operatorname{det} \mathscr{S}$ and $K_{N}=\operatorname{trace} \mathscr{S}$.

TheOrem ([8, Theorem 1.2]). Let $p \in S . \quad$ The following three conditions are equivalent.

(a) $p$ is an inflection point,

(b) $\mathscr{S}=0$ at $p$

(c) $\Delta_{p}=0$ and $K_{N}(p)=0$. 
Here, we give briefly another proof of the equivalence of (a) and (c). First, note that $p$ is an inflection point if and only if the equation (3) (and hence (4)) holds at $p$ for any $\theta$. Then, the equation (4) with $K_{N}=0$ implies that $\Lambda=0$, and hence that $\Delta=0$.

Moreover, we can get the following characterization in terms of $\Lambda$.

Lemma 6. A point $p$ in $S$ is an inflection point if and only if $\Lambda=0$ and $K_{N}=0$ at $p$. When $\boldsymbol{H}_{p} \neq \mathbf{0}$ especially, $p$ is an inflection point if and only if $\Lambda=0$ at $p$.

Proof. Set $\varphi=\varphi^{3} e_{3}+\varphi^{4} e_{4}$ and $h_{i j}=h_{i j}^{3} e_{3}+h_{i j}^{4} e_{4}$. Then, we obtain that $\varphi \wedge \boldsymbol{H}=\Lambda e_{3} \wedge e_{4}=\frac{1}{2} h_{11} \wedge h_{22}-\frac{\boldsymbol{i}}{2} h_{12} \wedge\left(h_{11}+h_{22}\right)$. Accordingly, the condition that $\Lambda=0$ is equivalent to that $h_{11} \wedge h_{22}=h_{12} \wedge\left(h_{11}+h_{22}\right)=0$. On the other hand, the condition that $K_{N}=0$ is equivalent to $h_{12} \wedge\left(h_{11}-h_{22}\right)=0$. Since the condition that $\mathscr{S}=0$ is equivalent to $h_{11} \wedge h_{22}=h_{12} \wedge h_{11}=h_{12} \wedge h_{22}=0$, we obtain the first assertion.

When $\boldsymbol{H} \neq \mathbf{0}, h_{12} \wedge\left(h_{11}+h_{22}\right)=0$ implies that there exists a real number $a$ satisfying $h_{12}=a\left(h_{11}+h_{22}\right)$. Then $h_{12} \wedge h_{11}=a h_{22} \wedge h_{11}=h_{22} \wedge h_{12}$. Hence the conditions $\boldsymbol{H} \neq \mathbf{0}$ and $\Lambda=0$ imply $h_{12} \wedge h_{11}=h_{12} \wedge h_{22}=0$. Therefore, we obtain the second assertion.

Remark 7. When $\boldsymbol{H}_{p}=\mathbf{0}$, it is clear that $p$ is an inflection point if and only if $K_{N}=0$ at $p$.

\section{Gauss maps}

Following Hoffman-Osserman [6], we will recall some terminologies.

Let $S$ be a connected Riemann surface and $X: S \rightarrow \mathbf{R}^{4}$ a conformal immersion. If $z=\xi+\boldsymbol{i} \eta$ is a local conformal parameter on $S$, the (conjugate) Gauss map $\bar{G}$ of $X$ is the map from $S$ into the complex quadric $Q_{2}$ in the complex projective 3 -space $\mathbf{C} P^{3}$ defined by

$$
\bar{G}(z)=\left[\frac{\partial X}{\partial z}\right] .
$$

$Q_{2}$ is biholomorphic to the product $S^{2} \times S^{2}$ of the Riemann sphere $S^{2}=\hat{\mathbf{C}}$. The identification $\hat{\mathbf{C}} \times \hat{\mathbf{C}} \cong Q_{2}$ is given by the map

$$
\begin{aligned}
& \varphi: \hat{\mathbf{C}} \times \hat{\mathbf{C}} \rightarrow Q_{2} \subset \mathbf{C} P^{3}, \\
& \left(w_{1}, w_{2}\right) \mapsto\left(1+w_{1} w_{2}, \boldsymbol{i}\left(1-w_{1} w_{2}\right), w_{1}-w_{2},-\boldsymbol{i}\left(w_{1}+w_{2}\right)\right) .
\end{aligned}
$$

Set $f_{k}=\pi_{k} \circ \bar{G}(k=1,2)$, where $\pi_{1}$ and $\pi_{2}$ are the projections from $Q_{2}$ on $S^{2}=\hat{\mathbf{C}}$. Then, the Gauss map $\bar{G}(z)$ is expressed by the pair $\left(f_{1}(z), f_{2}(z)\right)$ of the functions. 
Set $\Phi=\varphi\left(f_{1}, f_{2}\right)$ and

$$
A=\left(f_{2}-\overline{f_{1}},-\boldsymbol{i}\left(f_{2}+\overline{f_{1}}\right), 1+\overline{f_{1}} f_{2},-\boldsymbol{i}\left(1-\overline{f_{1}} f_{2}\right)\right) .
$$

We conclude that

$$
e_{1}=\sqrt{2} \frac{\operatorname{Re} \Phi}{\|\Phi\|}, \quad e_{2}=\sqrt{2} \frac{\operatorname{Im} \Phi}{\|\Phi\|}, \quad e_{3}=\sqrt{2} \frac{\operatorname{Re} \bar{A}}{\|A\|}, \quad e_{4}=\sqrt{2} \frac{\operatorname{Im} \bar{A}}{\|A\|}
$$

give an adapted local frame field on $S$ [6, Proposition 4.4]. It follows from $\bar{\Phi} \cdot \bar{A}=\bar{\Phi} \cdot A=0$ that

$$
\begin{aligned}
d\left(e_{1}\right. & \left.-\boldsymbol{i} e_{2}\right) \cdot\left(e_{3}+\boldsymbol{i} e_{4}\right) \wedge d\left(e_{1}-\boldsymbol{i} e_{2}\right) \cdot\left(e_{3}-i e_{4}\right) \\
& =\frac{4}{\|\Phi\|^{4}}(d \bar{\Phi} \cdot \bar{A}) \wedge(d \bar{\Phi} \cdot A) \\
& =\frac{4}{\|\Phi\|^{4}}\left\{\left(\bar{\Phi}_{z} \cdot \bar{A}\right)\left(\bar{\Phi}_{\bar{z}} \cdot A\right)-\left(\bar{\Phi}_{\bar{z}} \cdot \bar{A}\right)\left(\bar{\Phi}_{z} \cdot A\right)\right\} d z \wedge d \bar{z} \\
& =\frac{1}{\left(1+\left|f_{1}\right|^{2}\right)\left(1+\left|f_{2}\right|^{2}\right)} \overline{\left(f_{1 z} f_{2 z}-f_{1 z} f_{2 \bar{z}}\right)} d z \wedge d \bar{z} \\
& =\frac{\left(F_{1} \hat{F}_{2}-\hat{F}_{1} F_{2}\right)}{d z \wedge d \bar{z},}
\end{aligned}
$$

where

$$
F_{k}=F\left(f_{k}\right)=\frac{\left(f_{k}\right)_{\bar{z}}}{1+\left|f_{k}\right|^{2}}, \quad \text { and } \quad \hat{F}_{k}=\hat{F}\left(f_{k}\right)=\frac{\left(f_{k}\right)_{z}}{1+\left|f_{k}\right|^{2}}
$$

Denote the induced metric on $S$ by the form $d s^{2}=\lambda^{2}|d z|^{2}$. Then we obtain the following

LEMMA 8.

$$
\Lambda=\frac{i}{2 \lambda^{2}} \overline{\left(F_{1} \hat{F}_{2}-\hat{F}_{1} F_{2}\right)}
$$

Remark 9. The equation (1) combined with this lemma implies that

$$
4 \Delta=\left(K_{N}\right)^{2}-\frac{1}{\lambda^{4}}\left|F_{1} \hat{F}_{2}-\hat{F}_{1} F_{2}\right|^{2} .
$$

J. Monterde has also proved this equation in [10].

On the other hand, in [6, Proposition 4.5], it is also proved that the square norm of the mean curvature vector $\boldsymbol{H}$, the Gauss curvature $K$ and normal curvature $K_{N}$ of $X$ are given by 


$$
\begin{aligned}
|\boldsymbol{H}|^{2} & =\frac{2}{\lambda^{2}}\left(\left|F_{1}\right|^{2}+\left|F_{2}\right|^{2}\right), \\
K & =J_{1}+J_{2}, \\
K_{N} & =J_{1}-J_{2} .
\end{aligned}
$$

Here, $J_{k}(k=1,2)$ is the Jacobian of the map $f_{k}$ from $\left(S, \lambda^{2}|d z|^{2}\right)$ to the sphere $\left(S^{2}, g_{0}\right)$ of radius $1 / \sqrt{2}$ :

$$
J_{k}=\frac{2}{\lambda^{2}}\left(\left|F_{k}\right|^{2}-\left|\hat{F}_{k}\right|^{2}\right)
$$

\section{Inflection points}

In this section, we prove Theorem 1.

In order to prove that $X(S)$ in $\mathbf{R}^{4}$ lies in an affine 3 -space, we recall the following theorem for degenerate Gauss maps by Hoffman and Osserman [6]. A surface $M$ in $\mathbf{R}^{4}$ is said to have degenerate Gauss map if the image of $M$ under the Gauss map (5) lies in a hyperplane of $\mathbf{C} P^{3}$, that is, there exists a non-zero complex vector $B=\left(b_{1}, b_{2}, b_{3}, b_{4}\right)$ such that

$$
b_{1} \varphi_{1}(z)+b_{2} \varphi_{2}(z)+b_{3} \varphi_{3}(z)+b_{4} \varphi_{4}(z) \equiv 0,
$$

where $\left(\varphi_{1}(z), \varphi_{2}(z), \varphi_{3}(z), \varphi_{4}(z)\right)=\Phi(z)=\varphi\left(f_{1}(z), f_{2}(z)\right)$.

TheOREM 10 ([6, Theorem 5.3]). Let $M$ be a surface in $\mathbf{R}^{4}$ with degenerate Gauss map, so that (9) holds for some vector B. M lies in some affine 3-space in $\mathbf{R}^{4}$ if and only if $B$ can be chosen to be a real vector.

Proof of Theorem 1. First, if $K(p) \neq 0(p \in S)$, we show that the pullbacks of the metric $g_{0}$ on $S^{2}$ by $f_{1}, f_{2}$ induce a same metric $g$ on an open neighborhood around $p$ in $S$.

When $\boldsymbol{H}=\mathbf{0}$ at $p \in S$, from (6), we have $\left|F_{1}\right|=\left|F_{2}\right|=0$, and hence $\left(f_{1}\right)_{\bar{z}}=$ $\left(f_{2}\right)_{\bar{z}_{1}}=0$ at $p$. It follows from $K \neq 0$ and (7) that we have either $\left|\hat{F}_{1}\right| \neq 0$ or $\left|\hat{F}_{2}\right| \neq 0$ at $p$. Since $p$ is an inflection point, we have $K_{N}=0$ and, from (8), $\left|\hat{F}_{1}\right|=\left|\hat{F}_{2}\right|$, that is,

$$
\frac{\left|d f_{1}\right|^{2}}{\left(1+\left|f_{1}\right|^{2}\right)^{2}}=\frac{\left|d f_{2}\right|^{2}}{\left(1+\left|f_{2}\right|^{2}\right)^{2}} \neq 0 \quad \text { at } p \text {. }
$$

Now we consider the point $p$ at which $\boldsymbol{H} \neq \mathbf{0}$. Since $p$ is an inflection point, it follows from Lemmas 6 and 8 that

$$
F_{1} \hat{F}_{2}-\hat{F}_{1} F_{2}=0 \text {. }
$$

Since $\boldsymbol{H} \neq \mathbf{0}$, the equation (6) implies that $\left(F_{1}, F_{2}\right) \neq \mathbf{0}$. Hence, the equation (10) implies that there exists a complex number $\alpha$ such that at $p$

$$
\left(\begin{array}{c}
\hat{F}_{1} \\
\hat{F}_{2}
\end{array}\right)=\alpha\left(\begin{array}{c}
F_{1} \\
F_{2}
\end{array}\right) \text {. }
$$


Since $K \neq 0$, the equation (7) combined with (11) implies that $|\alpha| \neq 1$. Since $K_{N}=0$, it follows from $(8)$ and $|\alpha| \neq 1$ that

$$
\left|F_{1}\right|=\left|F_{2}\right| \neq 0 \text {. }
$$

This implies that $f_{1}, f_{2}$ are local diffeomorphisms. Moreover, we obtain that, for $k=1,2$,

$$
\begin{aligned}
& \frac{\left|d f_{k}\right|^{2}}{\left(1+\left|f_{k}\right|^{2}\right)^{2}} \\
& =\left|F_{k}\right|^{2}\left(\begin{array}{ll}
d \xi & d \eta
\end{array}\right)\left(\begin{array}{cc}
(1+\operatorname{Re}(\alpha))^{2}+\operatorname{Im}(\alpha)^{2} & -2 \operatorname{Im}(\alpha) \\
-2 \operatorname{Im}(\alpha) & (1-\operatorname{Re}(\alpha))^{2}+\operatorname{Im}(\alpha)^{2}
\end{array}\right)\left(\begin{array}{l}
d \xi \\
d \eta
\end{array}\right),
\end{aligned}
$$

where $z=\xi+i \eta$. Since $|\alpha| \neq 1$, these are nondegenerate.

Consequently, the pullbacks of the metric $g_{0}$ by $f_{1}, f_{2}$ induce a same metric $g$ on an open neighborhood around $p$ in $S$.

Second, we show that $\phi \circ f_{1}=f_{2}$ on $S$ for an orientation-preserving isometry $\phi \in \operatorname{Isom}_{+}\left(S^{2}, g_{0}\right)$. For any point $p \in S$, we can take $\phi_{p} \in \operatorname{Isom}_{+}\left(S^{2}, g_{0}\right)$ such that $\left(\phi_{p} \circ f_{1}\right)(p)=f_{2}(p)$ and $d\left(\phi_{p} \circ f_{1}\right)_{p}=\left(d f_{2}\right)_{p}$. From the above argument, there exist open neighborhoods $U_{p}$ in $S$ and $V_{p}$ in $S^{2}$ such that $\phi_{p} \circ f_{1}, f_{2}$ are isometric diffeomorphism from $\left(U_{p}, g\right)$ onto $\left(V_{p}, g_{0}\right)$. Hence $\phi_{p} \circ f_{1}=f_{2}$ on $U_{p}$ (e.g. [5, Lemma 11.2]).

For a fixed point $p_{0} \in S$, set $W=\left\{p \in S \mid\left(\phi_{p_{0}} \circ f_{1}\right)(p)=f_{2}(p)\right\}$. Then, $W$ is nonempty and obviously closed. Moreover, for any point $p \in W$, there exists a finite sequence of points $\left\{p_{k} \mid k=0, \ldots, n\right\}$ in $S$ such that $p_{n}=p$ and $U_{p_{k-1}} \cap U_{p_{k}} \neq \emptyset(k=1, \ldots, n)$. On $U_{p_{k-1}} \cap U_{p_{k}}$, we have $\phi_{p_{k-1}} \circ f_{1}=f_{2}=\phi_{p_{k}} \circ f_{1}$. Since $f_{1}\left(U_{p_{k-1}} \cap U_{p_{k}}\right) \subset\left(S^{2}, g_{0}\right)$ contains obviously at least three distinct points, then $\phi_{p_{k-1}}=\phi_{p_{k}}$. Then $\phi_{p}=\phi_{p_{0}}$, and hence $U_{p} \subset W$. This implies that $W$ is open. Since $S$ is connected, $W=S$, that is, $\phi_{p_{0}} \circ f_{1}=f_{2}$ on $S$.

The isometry $\phi_{p_{0}}$ can be expressed by

$$
\phi_{p_{0}}(w)=\frac{Q w-\bar{P}}{P w+\bar{Q}} \quad \text { for } w \in \hat{\mathbf{C}}=S^{2} \quad\left(P, Q \in \mathbf{C},|P|^{2}+|Q|^{2}=1\right) .
$$

Set $B=\left(b_{1}, b_{2}, b_{3}, b_{4}\right)=(-\operatorname{Re}(P), \operatorname{Im}(P), \operatorname{Re}(Q),-\operatorname{Im}(Q))$. Since $f_{2}=\phi_{p_{0}} \circ f_{1}=$ $\left(Q f_{1}-\bar{P}\right) /\left(P f_{1}+\bar{Q}\right)$, the Gauss map $\bar{G}=\varphi\left(f_{1}, f_{2}\right)$ of $X(S)$ satisfies the linear equation

$$
b_{1}\left(1+f_{1} f_{2}\right)+b_{2} \boldsymbol{i}\left(1-f_{1} f_{2}\right)+b_{3}\left(f_{1}-f_{2}\right)-b_{4} \boldsymbol{i}\left(f_{1}+f_{2}\right)=0 .
$$

Hence, the image of $\bar{G}$ is contained in the hyperplane in $\mathbf{C} P^{3}$, which is defined by the real vector $B$. Theorem 1 can now follow from Theorem 10 by Hoffman and Osserman. We then conclude that $X(S)$ lies in an affine 3-space in $\mathbf{R}^{4}$.

Remark 11. If all points of $S$ are inflection points, the dimension of the first normal space

$$
N_{1}^{X}(p)=\operatorname{span}\left\{\Pi(v, w) \mid v, w \in T_{p} S\right\}
$$


at any point $p \in S$ is less than 2. Assume that $N_{1}^{X}$ forms a rank-1 vector subbundle of the normal bundle $T^{\perp} S$. It is well known that $X(S)$ lies in an affine 3 -space in $\mathbf{R}^{4}$ if and only if $N_{1}^{X}$ is parallel in the normal connection of $X$ (see [2]). Moreover, from Theorem 1 in [3], if $N_{1}^{X}$ is nonparallel, we have that $K \equiv 0$.

\section{Examples}

Example 5.1 (Whitney sphere). Let $X$ be a conformal immersion from a Riemann sphere $\{(\cos u \cos v, \cos u \sin v, \sin u)\}$ into the complex 2-space $\mathbf{C}^{2} \cong \mathbf{R}^{4}$ given by

$$
\begin{aligned}
X(u, v) & =\left(\alpha(u) e^{i v}, \beta(u) e^{i v}\right) \\
& =(\alpha(u) \cos v, \alpha(u) \sin v, \beta(u) \cos v, \beta(u) \sin v),
\end{aligned}
$$

where

$$
\alpha(u)=\frac{\cos u}{1+\sin ^{2} u}, \quad \beta(u)=\frac{\cos u \sin u}{1+\sin ^{2} u} .
$$

The plane curve $\gamma_{a}(u)=(\alpha(u), \beta(u))$ is the lemniscate of Bernoulli, and $X$ gives the Whitney sphere. Following the computation in [1, Example 3.2], we have

$$
\begin{aligned}
& h^{3}=0, \quad h^{4}=\frac{-2 \sqrt{2} \cos u}{\sqrt{3-\cos 2 u}}, \\
& \varphi^{3}=-i \frac{\sqrt{2} \cos u}{\sqrt{3-\cos 2 u}}, \quad \varphi^{4}=\frac{-\sqrt{2} \cos u}{\sqrt{3-\cos 2 u}}, \\
& K_{N}=\frac{4 \cos ^{2} u}{3-\cos 2 u}, \quad \Lambda=i \frac{4 \cos ^{2} u}{3-\cos 2 u} .
\end{aligned}
$$

Hence, $X$ has only two infection points which are parabolic, and the other points are hyperbolic.

Example 5.2 (graphs in $\mathbf{R}^{4}$ ). For two functions $s(u, v)$ and $t(u, v)$, the graph surface $X(u, v)$ in $\mathbf{R}^{4}$ is given by

$$
X(u, v)=(u, v, s(u, v), t(u, v)) .
$$

Set

$$
\begin{aligned}
& E=X_{u} \cdot X_{u}=1+\left(s_{u}\right)^{2}+\left(t_{u}\right)^{2}, \quad F=X_{u} \cdot X_{v}=s_{u} s_{v}+t_{u} t_{v}, \\
& G=X_{v} \cdot X_{v}=1+\left(s_{v}\right)^{2}+\left(t_{v}\right)^{2}, \quad g=E G-F^{2}, \\
& n_{1}=\left(-s_{u},-s_{v}, 1,0\right), \quad n_{2}=\left(-t_{u},-t_{v}, 0,1\right), \\
& E^{\prime}=n_{1} \cdot n_{1}=1+\left(s_{u}\right)^{2}+\left(s_{v}\right)^{2}, \quad F^{\prime}=n_{1} \cdot n_{2}=s_{u} t_{u}+s_{v} t_{v}, \\
& G^{\prime}=n_{2} \cdot n_{2}=1+\left(t_{u}\right)^{2}+\left(t_{v}\right)^{2}, \quad g^{\prime}=E^{\prime} G^{\prime}-\left(F^{\prime}\right)^{2}
\end{aligned}
$$




$$
\begin{aligned}
& e_{1}=\frac{1}{\sqrt{E}} X_{u}, \quad e_{2}=\sqrt{\frac{E}{g}}\left(X_{v}-\frac{F}{E} X_{u}\right), \\
& e_{3}=\frac{1}{\sqrt{E^{\prime}}} n_{1}, \quad e_{4}=\sqrt{\frac{E^{\prime}}{g^{\prime}}}\left(n_{2}-\frac{F^{\prime}}{E^{\prime}} n_{1}\right) .
\end{aligned}
$$

Using the orthonormal frame $\left\{e_{1}, e_{2}, e_{3}, e_{4}\right\}$, we can compute the mean curvature vector $h^{3} e_{3}+h^{4} e_{4}$ and $K_{N}$ as follows:

$$
\begin{aligned}
& h^{\alpha}=\frac{1}{E} X_{u u} \cdot e_{\alpha}+\frac{E}{g}\left(X_{v v}-2 \frac{F}{E} X_{u v}+\frac{F^{2}}{E^{2}} X_{u u}\right) \cdot e_{\alpha} \quad(\alpha=3,4) \\
& K_{N}=\frac{1}{\sqrt{g^{\prime}}(\sqrt{g})^{3}}\left|\begin{array}{ccc}
E & F & G \\
s_{u u} & s_{u v} & s_{v v} \\
t_{u u} & t_{u v} & t_{v v}
\end{array}\right|, \\
& 2 \Lambda=\frac{\boldsymbol{i}}{\sqrt{g^{\prime}}(\sqrt{g})^{3}}\left|\begin{array}{ccc}
-E & \sqrt{g} \boldsymbol{i}-F & -\frac{1}{E}(\sqrt{g} \boldsymbol{i}-F)^{2} \\
s_{u u} & s_{u v} & s_{v v} \\
t_{u u} & t_{u v} & t_{v v}
\end{array}\right| .
\end{aligned}
$$

(i) For example, we set $s(u, v)=\frac{u^{2}}{2}+v$ and $t(u, v)=\frac{v^{2}}{2}+u$. Then, the graph surface has only hyperbolic points and no inflection point.

(ii) On the other hand, set $s=s(u)$ and $t=t(u)$. Then, we have that $K_{N} \equiv 0$ and $\Lambda \equiv 0$, and hence all points are inflection points. This graph is the product of a curve $(u, s(u), t(u))$ in $\mathbf{R}^{3}$ and a straight line in $\mathbf{R}^{4}$. Hence, the Gauss curvature $K$ is obviously identically zero. Therefore, this implies that the assertion in Theorem 1 never hold without an assumption on the Gauss curvature $K$.

\section{REFERENCES}

[1] R. Aiyama and K. Akutagawa, Semiumbilic points for minimal surfaces in Euclidean 4-space, to appear in Geom. Dedicata (2014).

[2] M. Dajczer et Al., Submanifolds and isometric immersions, Math. lecture series 13, Publish or Perish Inc., Houston, 1990.

[ 3 ] M. DajCZer And R. ToJeIro, Submanifolds with nonparallel first normal bundle, Canad. Math. Bull. 37 (1994), 330-337.

[ 4 ] R. A. Garcia, D. K. H. Mochida, M. D. C. R. Fuster and M. A. S. Ruas, Inflection points and topology of surfaces in 4-space, Trans. Amer. Math. Soc. 352 (2000), 3029-3043.

[ 5] S. Helgason, Differential geometry, Lie groups, and symmetric spaces, Academic Press, Inc., 1978.

[6] D. A. Hoffman and R. Osseerman, The Gauss map of surfaces in $\mathbf{R}^{3}$ and $\mathbf{R}^{4}$, Proc. London Math. Soc. 50 (1985), 27-56. 
[7] E. P. Lane, Projective differential geometry of curves and surfaces, University of Chicago Press, Chicago, 1932.

[ 8 ] J. A. LitTle, On singularities of submanifolds of higher dimensional Euclidean spaces, Ann. Math. Pura Appl., Ser. 4A. 83 (1969), 261-335.

[ 9 ] D. K. H. Mochida, M. D. C. R. Fuster and M. A. S. Ruas, The geometry of surfaces in 4-space from a contact viewpoint, Geometriae Dedicate 54 (1995), 323-332.

[10] J. Monterde, Construction of non-hyperspherical immersions, Bull. Braz. Math. Soc. New Series 43 (2012), 303-332.

[11] J. L. Weiner, The Gauss map for surfaces in 4-space, Math. Ann. 269 (1984), 541-560.

Reiko Aiyama

INSTITUTE OF MATHEMATICS

UNIVERSITY OF TSUKUBA

TsukuBa 305-8571

JAPAN

E-mail: aiyama@math.tsukuba.ac.jp

Kazuo Akutagawa

Department of Mathematics

Tokyo Institute of Technology

TOKYO 152-8551

JAPAN

E-mail: akutagawa@math.titech.ac.jp 